COMMENT. This study establishes the specific role of folic acid in the prevention of neural tube defects. Folic acid supplementation in a dose of $4 / \mathrm{mg} /$ day is now recommended for all women who have had a previously affected pregnancy, and the diet of all women who may bear children should contain an adequate amount of folic acid. An editorial in this issue of The Lancet comments that this supplement did not prevent all neural tube defects and six unsuccessful cases were not accounted for by unusually low serum folic acid concentrations. A heterogeneous aetiology for this group of disorders is suggested. The dosage of folic acid necessary to prevent neural tube defects is not entirely established, and the optimal duration of treatment before conception is uncertain. Can culturally appropriate dietary guidelines be prepared as a matter of urgency for all ethnic groups? Can the requisite folic acid be eaten in food instead of given as a supplement? Providing specific dietary advice about the prevention of malformations to women before pregnancy is a challenging public health problem.

\title{
EPILEPTIC SYNDROMES
}

\section{EPILEPTIC PALATAL MYOCLONUS}

A 14 year old girl with epilepsia partialis continua complicated by palatal myoclonus of focal cortical origin is reported from the Comprehensive Epilepsy Center, Graduate Hospital; and University of Pennsylvania School of Medicine, Philadelphia, PA. Her seizures consisted of staring, impairment of consciousness, and left facial twitching. Later she developed intermittent stuttering speech in association with palatal and lingual movements occurring up to 70 times a day. Several episodes of epilepsia partialis continua, lasting up to two weeks, with noted in the next three years. These were characterized by left facial and pharyngeal movements. They were refractory to medications. The EEG revealed theta and delta activity in the right central region with intermixed spikes, and a SPECT showed a focal area of increased perfusion in the right centrotemporal region. The epilepsia partialis continua remitted after several weeks. (Tatum WO et al. Epileptic palatal myoclonus. Neurology August 1991; $\underline{41}: 1305-1306)$.

COMMENT. The myoclonus epilepsies of childhood are divided into three major groups: (1) the progressive myoclonus epilepsies which include a number of degenerative and storage diseases; (2) the myoclonus epilepsies symptomatic of non-progressi $\%$ brain damage usually of prenatal or perinatal origin; (3) the cryptogenic myoclonus epilepsies (Aicardi J. Int Pediatr 1991; 6:195-200). More than 15 specific disorders may cause the progressive myoclonus epilepsy syndrome; reviewed by Berkovic SF et al. (J Clin Neurophysiol July 1991 ; $8: 261-274)$. The major causes of PME are the UnverrichtLundborg type, Lafora disease, neuronal ceroid lipofuscinoses, MERRF and sialidoses. Myoclonus is best controlled by valproate and/or clonaz:pam. 